\title{
Bifurcation Analysis of a Coupled Kuramoto-Sivashinsky- and Ginzburg-Landau-Type Model
}

\author{
Lei Shi ${ }^{1,2}$ \\ ${ }^{1}$ School of Sciences, Nanjing Agricultural University, Nanjing 210095, China \\ ${ }^{2}$ School of Mathematical Sciences and Jiangsu Key Laboratory for NSLSCS, Nanjing Normal University, Nanjing 210046, China
}

Correspondence should be addressed to Lei Shi; shileijsxh@163.com

Received 24 April 2013; Accepted 2 July 2013

Academic Editor: Abdul Hamid Kara

Copyright (C) 2013 Lei Shi. This is an open access article distributed under the Creative Commons Attribution License, which permits unrestricted use, distribution, and reproduction in any medium, provided the original work is properly cited.

\begin{abstract}
We study the bifurcation and stability of trivial stationary solution $(0,0)$ of coupled Kuramoto-Sivashinsky- and Ginzburg-Landautype equations (KS-GL) on a bounded domain $(0, L)$ with Neumann's boundary conditions. The asymptotic behavior of the trivial solution of the equations is considered. With the length $L$ of the domain regarded as bifurcation parameter, branches of nontrivial solutions are shown by using the perturbation method. Moreover, local behavior of these branches is studied, and the stability of the bifurcated solutions is analyzed as well.
\end{abstract}

\section{Introduction}

The mathematical theory of pattern formation [1-3] has a wide range of applications. In the field of fluid mechanics, Rayleigh-Bénard convection is the most widely studied example. Like the thermally driven Bénard convection, the surface tension-driven Marangoni convection is also an interesting pattern formation of nonlinear system. With mass transfer from liquid phase to gas phase, a typical setup for the Marangoni convection is a liquid layer resting on a rigid surface, with a free deformable upper interface contacting an infinite layer of gas. Nevertheless, at present, we still find it hard to analyze the governing equations for the Marangoni convection, that is, Navier-Stokes equations and mass (surfactant) transfer equation. What we can only use is numerical simulation so far. There is little work that has been done on the nonlinear Marangoni convection.

In this paper, we consider a simplified model, which is proposed to capture important nonlinear features yet more amendable to analyse:

$$
\begin{aligned}
& A_{t}=A+A_{x x}-\left|A^{2}\right| A+A h, \\
& h_{t}=-h_{x x}-h_{x x x x}+\alpha\left|A^{2}\right|_{x x},
\end{aligned}
$$

where $A(x, t)$ is the amplitude for the Marangoni convection mode and $h(x, t)$ is the interface deformation (real function). Equation (1) has been derived by Golovin et al. [4]. The constant $\alpha=k /\left(M a-M a_{c}\right)^{1 / 5}$ is positive, with $M a$ denoting the Marangoni number, and represents the gradient (derivative) of surface tension with respect to surfactant concentration. $M a_{c}$ is the critical Marangoni number at which the trivial stationary state becomes linearly unstable, and $k>0$ is a constant related to other system parameters.

Equation (1) without the interaction term $A h$ is the wellknown Ginzburg-Landau equation $[5,6]$, while the second equation without the term $\left|A^{2}\right|_{x x}$ is the linearized version of the so-called Kuramoto-Sivashinsky equation [7]. Both the G-L equation and K-S equation have been extensively investigated as model examples of infinite dimensional dynamical systems. In [8], Kazhdan et al. have done numerical simulation of this coupled system of Kuramoto-Sivashinsky- and Ginzburg-Landau-type equations (hereafter, KS-GL system). Duan et al. [9] have discussed the existence and uniqueness of global solutions of this coupled system, using the contraction mapping principle and energy estimates. Despite these publications on KS-GL equation, the static bifurcations of the equation have not been thoroughly studied. In this paper, we focus on studying bifurcations of the KS-GL system. In 
[10], Xiao and Gao analyzed the bifurcations of the 1D SwiftHohenberg equation with quintic nonlinearity. Two types of structures in the bifurcation diagrams are presented when the bifurcation points are closer, and their stabilities are analyzed. Li and Chen have applied singularity theory and the perturbation method to study the bifurcations of the 1D and $2 \mathrm{D}$ K-S equations and get the asymptotic expressions of the steady-state solution branches that have bifurcated from the equilibrium in $[11,12]$. In this paper, we will use the methods in [10-12] to discuss the bifurcated solutions. In (1), $A$ is complex. Namely, we can write $A=R \exp (i \theta)$. The additional phase $\theta$ makes analysis very complicated. Here, we restrict our attention to invariant subspace in which $A$ is real. We hope to return to the general case in future study. In this paper, we discuss steady solutions of the parabolic partial differential equations

$$
\begin{gathered}
A_{t}=A+A_{x x}-A^{3}+A h, \\
h_{t}=-h_{x x}-h_{x x x x}+\alpha\left(A^{2}\right)_{x x},
\end{gathered}
$$

on the cylindrical domain $Q=(0, L) \times R^{+}$subject to the boundary conditions

$$
A_{x}=h_{x}=A_{x x x}=h_{x x x}=0 \quad \text { at } x=0, L .
$$

The steady-state equation of (2) reads as

$$
\begin{gathered}
A+A_{x x}-A^{3}+A h=0, \quad 0<x<L, \\
-h_{x x}-h_{x x x x}+\alpha\left(A^{2}\right)_{x x}=0, \quad 0<x<L, \\
\frac{\partial A}{\partial x}=0, \quad \frac{\partial^{3} A}{\partial x^{3}}=0, \quad x=0, L, t>0, \\
\frac{\partial h}{\partial x}=0, \quad \frac{\partial^{3} h}{\partial x^{3}}=0, \quad x=0, L, t>0 .
\end{gathered}
$$

We will discuss the bifurcation of the trivial steady state of the equations.

\section{Analysis of the Trivial Steady State $(0,0)$}

In this section, we study some properties of the linear problem associated with problem (4). Let $X=\{(A, h) \in$ $H^{2}[0, L] \times H^{2}[0, L] \mid A^{\prime}(0)=A^{\prime}(L)=A^{\prime \prime \prime}(0)=A^{\prime \prime \prime}(L)=$ $\left.h^{\prime}(0)=h^{\prime}(L)=h^{\prime \prime \prime}(0)=h^{\prime \prime \prime}(L)=0\right\}$. We linearize the problem at the trivial solution $(0,0)$, and then we have the corresponding differential operator matrix

$$
\Gamma_{1}=\left(\begin{array}{cc}
\frac{\partial^{2}}{\partial x^{2}}+I & 0 \\
0 & -\frac{\partial^{2}}{\partial x^{2}}-\frac{\partial^{4}}{\partial x^{4}}
\end{array}\right)
$$

and the eigenvalue problem is

$$
\begin{gathered}
\varphi_{x x}+\varphi=\lambda \varphi, \quad 0<x<L, \\
-\psi_{x x}-\psi_{x x x x}=\lambda \psi, \quad 0<x<L, \\
\varphi_{x}(0)=\varphi_{x}(L)=\varphi_{x x x}(0)=\varphi_{x x x}(L)=0, \\
\psi_{x}(0)=\psi_{x}(L)=\psi_{x x x}(0)=\psi_{x x x}(L)=0 .
\end{gathered}
$$

The eigenvalues $\lambda$ are given by

$$
\begin{array}{r}
\lambda=\lambda_{m}=-\left(\frac{m \pi}{L}\right)^{2}+1, \quad \lambda=\delta_{n}=\left(\frac{n \pi}{L}\right)^{2}-\left(\frac{n \pi}{L}\right)^{4}, \\
n, m \in N .
\end{array}
$$

If $\lambda_{m}=\delta_{n}$, then the corresponding eigenvectors are

$$
\left(\begin{array}{c}
\cos \frac{m \pi x}{L} \\
0
\end{array}\right), \quad\left(\begin{array}{c}
0 \\
\cos \frac{n \pi x}{L}
\end{array}\right) \text {. }
$$

If $\lambda_{m} \neq \delta_{n}$, then for $\lambda_{m}=-(m \pi / L)^{2}+1$, the corresponding eigenvector is

$$
\left(\cos \frac{m \pi x}{L}\right)
$$

and for $\delta_{n}=(n \pi / L)^{2}-(n \pi / L)^{4}$, the corresponding eigenvector is

$$
\left(\begin{array}{c}
0 \\
\cos \frac{n \pi x}{L}
\end{array}\right)
$$

It will be convenient to rescale the spatial variable so that the domain $(0, L)$ maps onto the fixed domain $(0,1)$. Thus, we introduce the variables $\tilde{x}$ and $\tilde{u}, L \tilde{x}=x, \tilde{u}(\tilde{x})=u(x)$, $\tilde{v}(\tilde{x})=v(x)$, then omit the tildes, and we find that problem (4) becomes

$$
\begin{gathered}
A+L^{2} A_{x x}-L^{2} A^{3}+L^{2} A h=0, \quad 0<x<1, \\
-L^{2} h_{x x}-h_{x x x x}+\alpha L^{2}\left(A^{2}\right)_{x x}=0, \quad 0<x<1, \\
\frac{\partial A}{\partial x}=0, \quad \frac{\partial^{3} A}{\partial x^{3}}=0, \quad x=0,1, t>0, \\
\frac{\partial h}{\partial x}=0, \quad \frac{\partial^{3} h}{\partial x^{3}}=0, \quad x=0,1, t>0 .
\end{gathered}
$$

The corresponding eigenvalue problem at $(0,0)$ is

$$
\begin{gathered}
\varphi_{x x}+L^{2} \varphi=\lambda^{\prime} \varphi, \quad 0<x<1, \\
-L^{2} \psi_{x x}-\psi_{x x x x}=\lambda^{\prime} \psi, \quad 0<x<1, \\
\varphi_{x}(0)=\varphi_{x}(1)=\varphi_{x x x}(0)=\varphi_{x x x}(1)=0, \\
\psi_{x}(0)=\psi_{x}(1)=\psi_{x x x}(0)=\psi_{x x x}(1)=0 .
\end{gathered}
$$

It is easy to find that $\lambda^{\prime}=L^{2} \lambda$.

\section{Bifurcation and Stability Analysis in Different Cases}

Let $L$ be a bifurcation parameter; $L=\pi$ is a bifurcation point. In this section, we discuss how many nontrivial solution branches will be bifurcated from the trivial solution 
near $L=\pi$ and their asymptotic expression. Moreover, we will discuss the stability of the solution branches.

When $L=\pi$, the eigenvector at $(0,0)$ of $(12)$ is

$$
e_{1}=\left(\begin{array}{c}
\cos \pi x \\
c
\end{array}\right), \quad e_{2}=\left(\begin{array}{c}
0 \\
\cos \pi x
\end{array}\right), \quad c \in R
$$

We set

$$
\begin{gathered}
L=\pi+\gamma(\varepsilon) \cdot \varepsilon, \\
\left(\begin{array}{l}
A \\
h
\end{array}\right)=\sum_{i=1}^{n} \varepsilon \alpha_{i} e_{i}+\varepsilon^{2}\left(\begin{array}{l}
a(x, \varepsilon) \\
b(x, \varepsilon)
\end{array}\right),
\end{gathered}
$$

where $\varepsilon$ is a small parameter, $\left(\begin{array}{l}a(x, \varepsilon) \\ b(x, \varepsilon)\end{array}\right) \in\left\{e_{1}, e_{2}\right\}^{\perp}, \alpha_{1}^{2}+\alpha_{2}^{2}=1$.

Substituting (14), (15) into (11) leads to

$$
\begin{aligned}
\left(\varepsilon \alpha_{1} \cos \pi x+\varepsilon^{2} a(x, \varepsilon)\right)^{\prime \prime}+(\pi+\gamma(\varepsilon) \cdot \varepsilon)^{2} \\
\quad \times\left(\varepsilon \alpha_{1} \cos \pi x+\varepsilon^{2} a(x, \varepsilon)\right)-(\pi+\gamma(\varepsilon) \cdot \varepsilon)^{2} \\
\quad \times\left(\varepsilon \alpha_{1} \cos \pi x+\varepsilon^{2} a(x, \varepsilon)\right)^{3}+(\pi+\gamma(\varepsilon) \cdot \varepsilon)^{2} \\
\quad \times\left(\varepsilon \alpha_{1} \cos \pi x+\varepsilon^{2} a(x, \varepsilon)\right) \\
\quad \times\left(\varepsilon \alpha_{1} c+\varepsilon \alpha_{2} \cos \pi x+\varepsilon^{2} b(x, \varepsilon)\right)=0, \\
-(\pi+\gamma(\varepsilon) \cdot \varepsilon)^{2}\left(\varepsilon \alpha_{1} c+\varepsilon \alpha_{2} \cos \pi x+\varepsilon^{2} b(x, \varepsilon)\right)^{\prime \prime} \\
-\left(\varepsilon \alpha_{1} c+\varepsilon \alpha_{2} \cos \pi x+\varepsilon^{2} b(x, \varepsilon)\right)^{\prime \prime \prime \prime} \\
+\alpha(\pi+\gamma(\varepsilon) \cdot \varepsilon)^{2}\left(\left(\varepsilon \alpha_{1} \cos \pi x+\varepsilon^{2} a(x, \varepsilon)\right)^{2}\right)^{\prime \prime}=0,
\end{aligned}
$$

where ' means $\partial / \partial x$ and "means $\partial^{2} / \partial x^{2}$.

Letting the coefficient of $\varepsilon^{2}$ vanish in (16), (17) gives

$$
\begin{aligned}
a_{0}^{\prime \prime}(x) & +\pi^{2} a_{0}(x)+2 \pi \gamma(0) \alpha_{1} \cos \pi x \\
& +\pi^{2} \alpha_{1} \cos \pi x\left(\alpha_{1} c+\alpha_{2} \cos \pi x\right)=0, \\
b_{0}^{\prime \prime \prime \prime}(x) & +\pi^{2} b_{0}^{\prime \prime}(x)+2 \pi \gamma(0) \alpha_{1} \cos \pi x \\
& +\pi^{2} \alpha_{1} \cos \pi x\left(\alpha_{1} c+\alpha_{2} \cos \pi x\right)=0,
\end{aligned}
$$

where $\left(\begin{array}{l}a_{0}(x) \\ b_{0}(x)\end{array}\right)=\left(\begin{array}{l}a(x, 0) \\ b(x, 0)\end{array}\right) \in\left\{e_{1}, e_{2}\right\}^{\perp}$.

Taking the inner product of (16) with $\cos \pi x$, we obtain

$$
\begin{aligned}
& \int_{0}^{1} a_{0}^{\prime \prime}(x) \cos \pi x d x+\pi^{2} \int_{0}^{1} a_{0}(x) \cos \pi x d x \\
& \quad+2 \pi \gamma(0) \alpha_{1} \int_{0}^{1} \cos ^{2} \pi x d x \\
& +\pi^{2} \alpha_{1} \int_{0}^{1} \cos ^{2} \pi x\left(\alpha_{1} c+\alpha_{2} \cos \pi x\right) d x=0 .
\end{aligned}
$$

For the first term of (20), integrate it by part and from the boundary condition $a^{\prime}(0)=a^{\prime}(1)=0$, and we have

$$
\int_{0}^{1} a_{0}^{\prime \prime}(x) \cos \pi x d x=-\pi^{2} \int_{0}^{1} a_{0}(x) \cos \pi x d x .
$$

Substituting (21) to (20), we get

$$
\gamma(0)=-\frac{1}{2} c \alpha_{1} \pi, \quad\left(\alpha_{1} \neq 0\right)
$$

$\gamma(0)$ is an arbitrary constant, $\quad\left(\alpha_{1}=0\right)$.

From the aforementioned orthogonality condition, we know that

$$
\begin{gathered}
\int_{0}^{1} a_{0}(x) \cos \pi x+c b_{0}(x) d x=0 \\
\int_{0}^{1} b_{0}(x) \cos \pi x d x=0 .
\end{gathered}
$$

Taking the inner product of (19) with $\cos \pi x$, we obtain

$$
\begin{gathered}
\int_{0}^{1} b_{0}^{\prime \prime \prime \prime}(x) \cos \pi x d x+\pi^{2} \int_{0}^{1} b_{0}^{\prime \prime}(x) \cos \pi x d x \\
-2 \alpha_{2} \pi^{3} \gamma(0) \int_{0}^{1} \cos ^{2} \pi x \\
+2 \alpha \alpha_{1}^{2} \pi^{4} \int_{0}^{1} \cos 2 \pi x \cos \pi x d x=0 .
\end{gathered}
$$

Because of the boundary condition $b^{\prime}(0)=b^{\prime}(1)=b^{\prime \prime \prime}(0)=$ $b^{\prime \prime \prime}(1)=0$, orthogonality condition $(24)$, we get

$$
\gamma(0)=0, \quad\left(\alpha_{2} \neq 0\right)
$$

$\gamma(0)$ is an arbitrary constant, $\quad\left(\alpha_{2}=0\right)$,

from (25).

Comparing (22) and (26), for $\alpha_{1}^{2}+\alpha_{2}^{2}=1$, we deduce the following important relation:

$$
\gamma(0)=-\frac{1}{2} c \alpha_{1} \pi
$$

Substituting (27) to (18), we get

$$
a_{0}^{\prime \prime}(x)+\pi^{2} a_{0}(x)+\pi^{2} \alpha_{1} \alpha_{2} \cos ^{2} \pi x=0 .
$$

For $a_{0}(x)$ satisfies the boundary condition $a_{0}^{\prime}(0)=a_{1}^{\prime}(0)=$ $a_{0}^{\prime \prime \prime}(0)=a_{0}^{\prime \prime \prime}(1)$, we calculate from (28) that

$$
a_{0}(x)=k_{1} \cos \pi x+\frac{\alpha_{1} \alpha_{2}}{6} \cos 2 \pi x-\frac{\alpha_{1} \alpha_{2}}{2}, \quad k_{1} \in R .
$$

Next, we want to calculate $b_{0}(x)$ and substitute (27) to (19); we get the following ODE:

$$
\begin{gathered}
b_{0}^{\prime \prime \prime \prime}(x)+\pi^{2} b_{0}^{\prime \prime}(x)+c \alpha_{1} \alpha_{2} \pi^{4} \cos \pi x \\
+2 \alpha \alpha_{1}^{2} \pi^{4} \cos 2 \pi x=0 .
\end{gathered}
$$

By lengthy computations and the boundary conditions $b_{0}^{\prime}(0)=b_{1}^{\prime}(0)=b_{0}^{\prime \prime \prime}(0)=b_{0}^{\prime \prime \prime}(1)$, we deduce that

$$
\begin{gathered}
b_{0}(x)=c_{1}+c_{2} \cos \pi x-\frac{\alpha \alpha_{1}^{2}}{6} \cos 2 \pi x \\
c \alpha_{1} \alpha_{2}=0 .
\end{gathered}
$$


Taking the inner product with $\cos \pi x$ of (31), from (24), we know that

$$
\int_{0}^{1}\left(c_{1}+c_{2} \cos \pi x-\frac{\alpha \alpha_{1}^{2}}{6} \cos 2 \pi x\right) \cos \pi x d x=0,
$$

which gives us

$$
\begin{gathered}
c_{2}=0 \\
\left(\frac{\pi^{2}}{96} \alpha_{1}-\frac{\pi^{2}}{96} \alpha \alpha_{1}^{3}-\frac{\pi^{2}}{24} \alpha_{1}^{3}\right) \cos 3 \pi x b_{0}(x) \\
=c_{1}-\frac{\alpha \alpha_{1}^{2}}{6} \cos 2 \pi x
\end{gathered}
$$

Substituting (29), (35) to (23), we have

$$
\begin{aligned}
& \int_{0}^{1} \cos \pi x\left(k_{1} \cos \pi x+\frac{\alpha_{1} \alpha_{2}}{6} \cos 2 \pi x-\frac{\alpha_{1} \alpha_{2}}{2}\right) \\
& +c\left(c_{1}-\frac{\alpha \alpha_{1}^{2}}{6} \cos 2 \pi x\right) d x=0,
\end{aligned}
$$

from which we get

$$
\begin{gathered}
k_{1}=-2 c c_{1}, \\
a_{0}(x)=-2 c c_{1} \cos \pi x+\frac{\alpha_{1} \alpha_{2}}{6} \cos 2 \pi x-\frac{\alpha_{1} \alpha_{2}}{2} .
\end{gathered}
$$

From the previous discussion, we obtain

$$
c \alpha_{1} \alpha_{2}=0
$$

with the normal condition

$$
\alpha_{1}^{2}+\alpha_{2}^{2}-1=0
$$

When $c=0$, then we have

$$
\gamma_{0}=0
$$

at this time,

$$
\begin{gathered}
a_{0}(x)=\frac{\alpha_{1} \alpha_{2}}{6} \cos 2 \pi x-\frac{\alpha_{1} \alpha_{2}}{2}, \\
b_{0}(x)=c_{1}-\frac{\alpha \alpha_{1}^{2}}{6} \cos 2 \pi x .
\end{gathered}
$$

Letting the coefficient of $\varepsilon^{3}$ of (16), (17) vanish, we get

$$
\begin{aligned}
a_{1}^{\prime \prime}(x) & +\pi^{2} a_{1}(x)+2 \pi \alpha_{1} \gamma_{1}(0) \cos \pi x \\
& -\pi^{2} \alpha_{1}^{3} \cos ^{3} \pi x+\pi^{2} \alpha_{1} \cos \pi x b_{0}(x) \\
& +\pi^{2} \alpha_{2} a_{0}(x) \cos \pi x=0, \\
b_{1}^{\prime \prime \prime \prime}(x) & +\pi^{2} b_{1}^{\prime \prime}(x)-2 \alpha_{1} \alpha \pi^{2} \cos \pi x a_{0}^{\prime \prime}(x) \\
& +4 \alpha_{1} \alpha \pi^{3} \sin \pi x a_{0}^{\prime}(x) \\
& +2 \alpha_{1} \alpha \pi^{4} \cos \pi x a_{0}(x) \\
& -2 \pi^{3} \gamma_{1}(0) \alpha_{2} \cos \pi x=0
\end{aligned}
$$

where $a_{1}(x)=\left.(\partial a(x, \varepsilon) / \partial x)\right|_{\varepsilon=0}, b_{1}(x)=\left.(\partial b(x, \varepsilon) / \partial x)\right|_{\varepsilon=0}$ and $\gamma_{1}(0)=\gamma^{\prime}(0)$.

Taking the inner product of (42) with $\cos \pi x$, we obtain

$$
\begin{aligned}
& \int_{0}^{1} a_{1}^{\prime \prime}(x) \cos \pi x d x+\pi^{2} \int_{0}^{1} a_{1}(x) \cos \pi x d x \\
& \quad+2 \pi \alpha_{1} \gamma_{1}(0) \int_{0}^{1} \cos ^{2} \pi x d x-\pi^{2} \alpha_{1}^{3} \int_{0}^{1} \cos ^{4} \pi x d x \\
& \quad+\pi^{2} \alpha_{1} \int_{0}^{1} \cos ^{2} \pi x b_{0}(x) d x \\
& +\pi^{2} \alpha_{2} \int_{0}^{1} a_{0}(x) \cos ^{2} \pi x d x=0,
\end{aligned}
$$

from which we know that

$$
\gamma_{1}(0)=\frac{3}{8} \pi \alpha_{1}^{2}+\frac{\alpha \alpha_{1}^{2} \pi}{24}-\frac{c_{1} \pi}{2}+\frac{5}{24} \pi \alpha_{2}^{2}, \quad \alpha_{1} \neq 0,
$$

$$
\gamma_{1}(0) \text { is an arbitrary constant, } \quad \alpha_{1}=0 .
$$

Next, we discuss the expression and the stability of the bifurcated solutions in different cases.

Case 1. $c=0, \alpha_{1} \neq 0$.

Substituting (41), (45) to (42), we have

$$
a_{1}^{\prime \prime}(x)+\pi^{2} a_{1}(x)+\left(\frac{\pi^{2}}{12} \alpha_{1}-\frac{\pi^{2}}{12} \alpha \alpha_{1}^{3}-\frac{\pi^{2}}{3} \alpha_{1}^{3}\right) \cos 3 \pi x=0 \text {. }
$$

Because of the orthogonality condition

$$
\int_{0}^{1} a_{1}(x) \cos \pi x d x=0,
$$

and boundary condition

$$
a_{1}^{\prime}(0)=a_{1}^{\prime}(1)=0
$$

we get

$$
a_{1}(x)=\left(\frac{\pi^{2}}{96} \alpha_{1}-\frac{\pi^{2}}{96} \alpha \alpha_{1}^{3}-\frac{\pi^{2}}{24} \alpha_{1}^{3}\right) \cos 3 \pi x .
$$

Substituting (41) to (43), we have

$$
\begin{aligned}
b_{1}^{\prime \prime \prime \prime}(x) & +\pi^{2} b_{1}^{\prime \prime}(x) \\
& -\left(2 \pi^{3} \gamma_{1}(0) \alpha_{2}+\frac{5}{6} \alpha \alpha_{1}^{2} \alpha_{2} \pi^{4}\right) \cos \pi x \\
+ & \frac{3}{2} \alpha \alpha_{1}^{2} \alpha_{2} \pi^{4} \cos 3 \pi x=0 .
\end{aligned}
$$

Taking the inner product of (50) with $\cos \pi x$, we obtain

$$
\begin{gathered}
\int_{0}^{1} b_{1}^{\prime \prime \prime \prime}(x) \cos \pi x d x+\pi^{2} \int_{0}^{1} b_{1}^{\prime \prime}(x) \cos \pi x d x \\
-\left(2 \pi^{3} \gamma_{1}(0) \alpha_{2}+\frac{5}{6} \alpha \alpha_{1}^{2} \alpha_{2} \pi^{4}\right) \int_{0}^{1} \cos ^{2} \pi x d x \\
+\frac{3}{2} \alpha \alpha_{1}^{2} \alpha_{2} \pi^{4} \int_{0}^{1} \cos 3 \pi x \cos \pi x d x=0,
\end{gathered}
$$


for $b_{1}(x)$ satisfies the orthogonality condition

$$
\int_{0}^{1} b_{1}(x) \cos \pi x d x=0
$$

and the boundary condition

$$
b_{1}^{\prime}(0)=b_{1}^{\prime}(1)=b_{1}^{\prime \prime \prime}(0)=b_{1}^{\prime \prime \prime}(1) ;
$$

we know

$$
\int_{0}^{1} b_{1}^{\prime \prime \prime \prime}(x) \cos \pi x d x+\pi^{2} \int_{0}^{1} b_{1}^{\prime \prime}(x) \cos \pi x d x=0,
$$

which forces

$$
2 \pi^{3} \gamma_{1}(0) \alpha_{2}+\frac{5}{6} \alpha \alpha_{1}^{2} \alpha_{2} \pi^{4}=0
$$

From (45) and (55), we get

$$
\begin{array}{r}
\gamma_{1}(0)=-\frac{5}{12} \alpha \alpha_{1}^{2} \pi=\frac{3}{8} \pi \alpha_{1}^{2}+\frac{\alpha \alpha_{1}^{2} \pi}{24}-\frac{c_{1} \pi}{2}+\frac{5}{24} \pi \alpha_{2}^{2}, \\
\alpha_{2} \neq 0,
\end{array}
$$

or

$$
\begin{array}{r}
\gamma_{1}(0)=\frac{3}{8} \pi \alpha_{1}^{2}+\frac{\alpha \alpha_{1}^{2} \pi}{24}-\frac{c_{1} \pi}{2}+\frac{5}{24} \pi \alpha_{2}^{2}, \\
\alpha_{2}=0 .
\end{array}
$$

Substituting (55) to (50), we get

$$
b_{1}^{\prime \prime \prime \prime}(x)+\pi^{2} b_{1}^{\prime \prime}(x)+\frac{3}{2} \alpha \alpha_{1}^{2} \alpha_{2} \pi^{4} \cos 3 \pi x=0,
$$

which gives us

$$
b_{1}(x)=\widetilde{c_{1}}-\frac{1}{48} \alpha \alpha_{1}^{2} \alpha_{2} \cos 3 \pi x .
$$

As has been discussed previously, we have the following.

For $L$ near $\pi$, there are nontrivial steady-state solution branches of (11) bifurcated from the trivial solution:

$$
\begin{aligned}
A(\varepsilon)= & \varepsilon \alpha_{1} \cos \pi x+\varepsilon^{2}\left(\frac{\alpha_{1} \alpha_{2}}{6} \cos \pi x-\frac{\alpha_{1} \alpha_{2}}{2}\right) \\
& +\varepsilon^{3}\left(\frac{\pi^{2}}{96} \alpha_{1}-\frac{\pi^{2}}{96} \alpha \alpha_{1}^{3}-\frac{\pi^{2}}{24} \alpha_{1}^{3}\right) \cos 3 \pi x \\
& +o\left(\varepsilon^{3}\right), \\
h(\varepsilon)= & \varepsilon \alpha_{2} \cos \pi x+\varepsilon^{2}\left(c_{1}-\frac{\alpha \alpha_{1}^{2}}{6} \cos 2 \pi x\right) \\
& +\varepsilon^{3}\left(\widetilde{c_{1}}-\frac{1}{48} \alpha \alpha_{1}^{2} \alpha_{2} \cos 3 \pi x\right)+o\left(\varepsilon^{3}\right), \\
& L=\pi+\varepsilon^{2} \gamma_{1}(0)+o\left(\varepsilon^{2}\right) .
\end{aligned}
$$

$\alpha_{1} \neq 0, \alpha_{2}, \gamma_{1}(0)$ satisfy (56), (57). $c_{1}, \widetilde{c_{1}} \in R . c_{1}, \widetilde{c_{1}}$ are arbitrary constants.

Next, we wish to consider the stability of the nontrivial solutions given in (60).

Considering the eigenvalue problem

$$
F_{A, h}(A(\varepsilon), h(\varepsilon), L(\varepsilon))\left(\begin{array}{l}
\phi(\varepsilon) \\
\psi(\varepsilon)
\end{array}\right)=\lambda(\varepsilon)\left(\begin{array}{l}
\phi(\varepsilon) \\
\psi(\varepsilon)
\end{array}\right)
$$

since $\lambda(0)=0, \phi(0)=\cos \pi x, \psi(0)=0$, we assume

$$
\begin{gathered}
\left(\begin{array}{l}
\phi(\varepsilon) \\
\psi(\varepsilon)
\end{array}\right)=\left(\begin{array}{c}
\cos \pi x \\
0
\end{array}\right)+\varepsilon\left(\begin{array}{l}
\phi_{1} \\
\psi_{1}
\end{array}\right)+\varepsilon^{2}\left(\begin{array}{l}
\phi_{2} \\
\psi_{2}
\end{array}\right)+\cdots, \\
\lambda(\varepsilon)=\lambda_{1} \varepsilon+\lambda_{2} \varepsilon^{2}+\cdots, \\
F_{A, h}(A(\varepsilon), h(\varepsilon), L(\varepsilon)) \\
=\left(\begin{array}{c}
L^{2}+\frac{\partial^{2}}{\partial x^{2}}-3 L^{2} A^{2}(\varepsilon)+L^{2} h(\varepsilon) \\
2 \alpha L^{2}\left(2 \frac{\partial A(\varepsilon)}{\partial x} \frac{\partial}{\partial x}+\frac{\partial^{2} A(\varepsilon)}{\partial x^{2}}+A(\varepsilon) \frac{\partial^{2}}{\partial x^{2}}\right)-L^{2} \frac{\partial^{2}}{\partial x^{2}}-\frac{\partial^{4}}{\partial x^{4}}
\end{array}\right) .
\end{gathered}
$$

Substituting (62) to (61), we get

$$
\begin{aligned}
\frac{\partial^{2}}{\partial x^{2}} \phi & (\varepsilon)+L^{2} \phi(\varepsilon)-3 L^{2} A^{2}(\varepsilon) \phi(\varepsilon) \\
& +L^{2} h(\varepsilon) \phi(\varepsilon)+L^{2} A(\varepsilon) \psi(\varepsilon) \\
& -\lambda(\varepsilon) \phi(\varepsilon)=0, \\
2 \alpha L^{2} & \left(2 \frac{\partial A(\varepsilon)}{\partial x} \frac{\partial \phi(\varepsilon)}{\partial x}+\frac{\partial^{2} A(\varepsilon)}{\partial x^{2}} \phi(\varepsilon)+A(\varepsilon) \frac{\partial^{2} \phi(\varepsilon)}{\partial x^{2}}\right) \\
& -L^{2} \frac{\partial^{2} \psi}{\partial x^{2}}-\frac{\partial^{4} \psi}{\partial x^{4}}-\lambda(\varepsilon) \psi(\varepsilon)=0 .
\end{aligned}
$$

On the branches given in (60), equating the coefficient of $\varepsilon$ in (63) to 0 , we obtain

$$
\begin{gathered}
\phi_{1}^{\prime \prime}+\pi^{2} \phi_{1}+\alpha_{2} \pi^{2} \cos ^{2} \pi x-\lambda_{1} \cos \pi x=0, \\
4 \alpha \alpha_{1} \pi^{4} \cos 2 \pi x+\pi^{2} \psi_{1}^{\prime \prime}+\psi_{1}^{\prime \prime \prime \prime}=0 .
\end{gathered}
$$

From (64) and the boundary conditions

$$
\begin{aligned}
& \phi_{1}^{\prime}(0)=\phi_{1}^{\prime}(1)=\phi_{1}^{\prime \prime \prime}(0)=\phi_{1}^{\prime \prime \prime}(1), \\
& \psi_{1}^{\prime}(0)=\psi_{1}^{\prime}(1)=\psi_{1}^{\prime \prime \prime}(0)=\psi_{1}^{\prime \prime \prime}(1),
\end{aligned}
$$

we get

$$
\begin{gathered}
\lambda_{1}=0, \\
\left(\begin{array}{l}
\phi_{1} \\
\psi_{1}
\end{array}\right)=\left(\begin{array}{c}
p_{1} \cos \pi x+\frac{\alpha_{2}}{6} \cos 2 \pi x-\frac{\alpha_{2}}{2} \\
q_{1}+q_{2} \cos \pi x-\frac{\alpha \alpha_{1}}{3} \cos 2 \pi x
\end{array}\right) \cdot p_{1}, \\
q_{1}, q_{2} \in R .
\end{gathered}
$$


Using the second-order term of $\varepsilon$ in (63), we obtain

$$
\begin{aligned}
\phi_{2}^{\prime \prime}+ & \pi^{2} \phi_{2}-\left[\left(-\frac{3}{2} \alpha_{1}^{2}+\alpha_{1} q_{1}-\frac{\alpha}{6} \alpha_{1}^{2}\right) \pi^{2}-\lambda_{2}\right] \cos \pi x \\
& +\left(\frac{p_{1} \alpha_{2}}{2}+\frac{q_{2} \alpha_{1}}{2}\right) \pi^{2} \cos 2 \pi x \\
& +\left(-\frac{3}{4} \alpha_{1}^{2}+\frac{1}{12} \alpha_{1}^{2}-\frac{\pi^{2}}{4} \alpha \alpha_{1}^{2}\right) \cos 3 \pi x \\
& +\frac{\pi^{2}}{2} p_{1} \alpha_{2}+\frac{\pi^{2}}{2} q_{2} \alpha_{1}=0, \\
\psi_{2}^{\prime \prime \prime \prime}+ & \pi^{2} \psi_{2}^{\prime \prime}-\frac{5}{3} \alpha_{1} \alpha_{2} \alpha \pi^{4} \cos \pi x+4 \alpha \alpha_{1} p_{1} \pi^{4} \cos 2 \pi x \\
& +3 \alpha_{1} \alpha_{2} \alpha \pi^{4} \cos 3 \pi x=0,
\end{aligned}
$$

and considering the boundary condition

$$
\begin{aligned}
& \phi_{2}^{\prime}(0)=\phi_{2}^{\prime}(1)=\phi_{1}^{\prime \prime \prime}(0)=\phi_{2}^{\prime \prime \prime}(1), \\
& \psi_{2}^{\prime}(0)=\psi_{2}^{\prime}(1)=\psi_{2}^{\prime \prime \prime}(0)=\psi_{2}^{\prime \prime \prime}(1),
\end{aligned}
$$

we deduce that (67) has solution if and only if $\alpha_{2}=0$; in this case, we have $\alpha_{1}= \pm 1\left(\alpha_{1}^{2}+\alpha_{2}^{2}=1\right)$. Taking the sign of $\varepsilon$ into account in (15), we consider $\alpha_{1}=1$ only. Therefore, we have

$$
\lambda_{2}=\left(-\frac{3}{2}-\frac{\alpha}{6}+q_{1}\right) \pi^{2}
$$

$\left(\begin{array}{l}\phi_{2} \\ \psi_{2}\end{array}\right)$

$$
=\left(\begin{array}{c}
p_{2} \cos \pi x+\frac{q_{2}}{6} \cos 2 \pi x-\left(\frac{3}{32}+\frac{\alpha}{32}\right) \cos 3 \pi x-\frac{q_{2}}{2} \\
\widetilde{q_{1}}+\widetilde{q_{2}} \cos \pi x-\frac{p_{1} \alpha}{3} \cos 2 \pi x
\end{array}\right) \cdot p_{2},
$$

So in Case 1, on the branches (60), the eigenvalue of $F_{A, h}(A(\varepsilon), h(\varepsilon), L(\varepsilon))$ is

$$
\lambda(\varepsilon)=\left(-\frac{3}{2}-\frac{\alpha}{6}+q_{1}\right) \pi^{2} \varepsilon^{2}+o\left(\varepsilon^{2}\right) .
$$

The corresponding eigenfunction is

$$
\begin{aligned}
\left(\begin{array}{l}
\phi \\
\psi
\end{array}\right)= & \left(\begin{array}{c}
\cos \pi x \\
0
\end{array}\right)+\left(\begin{array}{c}
p_{1} \cos \pi x \\
q_{1}+q_{2} \cos \pi x-\frac{\alpha}{3} \cos 2 \pi x
\end{array}\right) \varepsilon \\
& +\left(\begin{array}{c}
p_{2} \cos \pi x-\left(\frac{3}{32}+\frac{\alpha}{32}\right) \cos 3 \pi x-\frac{q_{2}}{2}+\frac{q_{2}}{6} \cos 2 \pi x \\
\widetilde{q_{1}}+\widetilde{q_{2}} \cos \pi x-\frac{p_{1} \alpha}{3} \cos 2 \pi x
\end{array}\right) \varepsilon^{2} \\
& +o\left(\varepsilon^{2}\right) .
\end{aligned}
$$

From (70), we know that the eigenvalue of the linearized operator $F_{A, h}(A(\varepsilon), h(\varepsilon), L(\varepsilon))$ at the nontrivial solutions given in (60) is negative if $q_{1}<(3 / 2+\alpha / 6)$, and positive if $q_{1}>(3 / 2+\alpha / 6)$. Finally, for small $\varepsilon$, if $q_{1}<(3 / 2+\alpha / 6)$, then the corresponding solution branches are stable; if $q_{1}>$ $(3 / 2+\alpha / 6)$, then the corresponding solution branches are unstable; if $q_{1}=(3 / 2+\alpha / 6)$, we need higher-order items of $\lambda$ to determine the result.

Thus, we have proved the following theorems.

Theorem 1. For $L$ near $\pi$, there are nontrivial steady-state solution branches of (4) bifurcated from the trivial solution:

$$
\begin{gathered}
A(\varepsilon)=\varepsilon \alpha_{1} \cos \frac{\pi x}{L}+\varepsilon^{2}\left(\frac{\alpha_{1} \alpha_{2}}{6} \cos \frac{2 \pi x}{L}-\frac{\alpha_{1} \alpha_{2}}{2}\right) \\
+\varepsilon^{3}\left(\frac{\pi^{2}}{96} \alpha_{1}-\frac{\pi^{2}}{96} \alpha \alpha_{1}^{3}-\frac{\pi^{2}}{24} \alpha_{1}^{3}\right) \cos \frac{3 \pi x}{L}+o\left(\varepsilon^{3}\right) \\
h(\varepsilon)=\varepsilon \alpha_{2} \cos \frac{\pi x}{L}+\varepsilon^{2}\left(c_{1}-\frac{\alpha \alpha_{1}^{2}}{6} \cos \frac{2 \pi x}{L}\right) \\
+\varepsilon^{3}\left(\widetilde{c_{1}}-\frac{1}{48} \alpha \alpha_{1}^{2} \alpha_{2} \cos 3 \pi x\right)+o\left(\varepsilon^{3}\right) \\
L=\pi+\varepsilon^{2} \gamma_{1}(0)+o\left(\varepsilon^{2}\right)
\end{gathered}
$$

where $\alpha_{1}=1, \alpha_{2}=0, \gamma_{1}(0)=3 / 8+\alpha / 24-c_{1} / 2, c_{1}, \widetilde{c_{1}} \in R$.

Remark 2. Since $\gamma_{1}(0)=3 / 8+\alpha / 24-c_{1} / 2$, when $c_{1}<3 / 4+$ $\alpha / 12, L=\pi$ is a supercritical bifurcation point; when $c_{1}>$ $(3 / 4+\alpha / 12), L=\pi$ is a supercritical bifurcation point.

Theorem 3. The eigenvalue of the linearized operator $F_{A, h}(A(\varepsilon), h(\varepsilon), L(\varepsilon))$ at the nontrivial solutions given in Theorem 1 is negative if $q_{1}<(3 / 2+\alpha / 6)$, positive if $q_{1}>(3 / 2+$ $\alpha / 6)$. Finally, for small $\varepsilon$, if $q_{1}<(3 / 2+\alpha / 6)$, then the corresponding solution branches are stable; if $q_{1}>(3 / 2+\alpha / 6)$, then the corresponding solution branches are unstable.

Case 2. $c=0, \alpha_{1}=0$.

If $c=0, \alpha_{1}=0$, then $\alpha_{2}= \pm 1$; taking the sign of $\varepsilon$ into account in (15), we consider $\alpha_{2}=1$ only. In this case, from (40), (41), (45), (49), (56), and (59), we have

$$
\begin{array}{lll}
a_{0}(x)=0, & b_{0}(x)=c_{1}, & \gamma(0)=0, \\
a_{1}(x)=0, & b_{1}(x)=\widetilde{c_{1}}, & \gamma_{1}(0)=0 .
\end{array}
$$

Similarly discussed as in Case 1 previously, we get

$$
\gamma_{2}(0)=\gamma_{3}(0)=\gamma_{4}(0)=\cdots=0
$$

Remark 4. Since $\gamma(0)=\gamma_{1}(0)=\gamma_{2}(0)=\gamma_{3}(0)=\gamma_{4}(0)=$ $\cdots=0$, there are no solution branches of (4) bifurcated from the trivial solution in Case 2.

Case 3. $c \neq 0, \alpha_{1} \neq 0$.

If $c \neq 0, \alpha_{1} \neq 0$, then $\alpha_{2}=0, \alpha_{1}= \pm 1$; taking the sign of $\varepsilon$ into account in (15), we consider $\alpha_{2}=1$ only. In this case, 
from (27), (31), and (37), we have

$$
\begin{gathered}
a_{0}(x)=-2 c c_{1} \cos \pi x, \\
b_{0}(x)=c_{1}-\frac{\alpha}{6} \cos 2 \pi x, \\
\gamma(0)=-\frac{1}{2} c \pi .
\end{gathered}
$$

Letting the coefficient of $\varepsilon^{3}$ of (16) vanish, we get

$$
\begin{gathered}
a_{1}^{\prime \prime}(x)+\pi^{2} a_{1}(x)+\left(c_{1} \pi^{2}+2 \pi \gamma_{1}-\frac{3}{4} c^{2} \pi^{2}\right. \\
\left.-\frac{3}{4} \pi^{2}-\frac{\alpha}{12} \pi^{2}\right) \cos \pi x \\
+\left(\frac{\alpha}{12} \pi^{2}-\frac{\pi^{2}}{4}\right) \cos 3 \pi x=0 .
\end{gathered}
$$

Taking the inner product of (76) with $\cos \pi x$, we obtain

$$
\begin{gathered}
\int_{0}^{1} a_{1}^{\prime \prime}(x) \cos \pi x d x+\pi^{2} \int_{0}^{1} a_{1}(x) \cos \pi x d x \\
+\int_{0}^{1}\left(c_{1} \pi^{2}+2 \pi \gamma_{1}-\frac{3}{4} c^{2} \pi^{2}\right. \\
\left.-\frac{3}{4} \pi^{2}-\frac{\alpha}{12} \pi^{2}\right) \cos ^{2} \pi x d x \\
+\left(\frac{\alpha}{12} \pi^{2}-\frac{\pi^{2}}{4}\right) \int_{0}^{1} \cos 3 \pi x \cos \pi x d x
\end{gathered}
$$

from which we get

$$
\begin{gathered}
\gamma_{1}(0)=\frac{3}{8} c^{2} \pi+\frac{3}{8} \pi-\frac{c_{1} \pi}{2}+\frac{\alpha}{24} \pi, \\
a_{1}(x)=k_{2} \cos \pi x+\left(\frac{\alpha}{96}-\frac{1}{32}\right) \cos 3 \pi x .
\end{gathered}
$$

Letting the coefficient of $\varepsilon^{3}$ of (17) vanish, we get

$$
b_{1}^{\prime \prime \prime \prime}(x)+\pi^{2} b_{1}^{\prime \prime}(x)-\left(8 c_{1}+\frac{8}{3}\right) \pi^{4} \alpha c \cos 2 \pi x=0,
$$

from which we get

$$
b_{1}(x)=\left(\frac{2}{3} c_{1} \alpha c+\frac{2}{9} \alpha c\right) \cos 2 \pi x+c_{2}
$$

Substituting (78), (80) to the orthogonality condition

$$
\int_{0}^{1} \cos \pi x a_{1}(x)+c b_{1}(x) d x=0
$$

we get

$$
k_{2}=-2 c c_{2}
$$

Thus, we have that for $L$ near $\pi$, there are nontrivial steadystate solution branches of (11) bifurcated from the trivial solution:

$$
\begin{aligned}
A(\varepsilon)= & \varepsilon \cos \pi x+\varepsilon^{2}\left(-2 c c_{1}\right) \cos \pi x \\
& +\varepsilon^{3}\left[-2 c c_{2} \cos \pi x+\left(\frac{\alpha}{96}-\frac{1}{32}\right) \cos 3 \pi x\right]+o\left(\varepsilon^{3}\right), \\
h(\varepsilon)= & \varepsilon^{2}\left(c_{1}-\frac{\alpha}{6} \cos 2 \pi x\right) \\
& +\varepsilon^{3}\left(c_{2}+\frac{2}{3} c_{1} \alpha c+\frac{2}{9} \alpha c\right) \cos 2 \pi x+o\left(\varepsilon^{3}\right), \\
L= & \pi-\frac{1}{2} c \pi \varepsilon+\left(\frac{3}{8} c^{2} \pi+\frac{3}{8} \pi\right. \\
& \left.\quad-\frac{c_{1} \pi}{2}+\frac{\alpha}{24} \pi\right) \varepsilon^{2}+o\left(\varepsilon^{2}\right) .
\end{aligned}
$$

Next, we wish to consider the stability of the nontrivial solutions given in (83).

Considering the eigenvalue problem

$$
F_{A, h}(A(\varepsilon), h(\varepsilon), L(\varepsilon))\left(\begin{array}{c}
\phi(\varepsilon) \\
\psi(\varepsilon)
\end{array}\right)=\lambda(\varepsilon)\left(\begin{array}{c}
\phi(\varepsilon) \\
\psi(\varepsilon)
\end{array}\right),
$$

since $\lambda(0)=0, \phi(0)=\cos \pi x, \psi(0)=c$, we assume that

$$
\begin{gathered}
\left(\begin{array}{l}
\phi(\varepsilon) \\
\psi(\varepsilon)
\end{array}\right)=\left(\begin{array}{c}
\cos \pi x \\
c
\end{array}\right)+\varepsilon\left(\begin{array}{l}
\phi_{1} \\
\psi_{1}
\end{array}\right)+\varepsilon^{2}\left(\begin{array}{l}
\phi_{2} \\
\psi_{2}
\end{array}\right)+\cdots \\
\lambda(\varepsilon)=\lambda_{1} \varepsilon+\lambda_{2} \varepsilon^{2}+\cdots
\end{gathered}
$$

$F_{A, h}(A(\varepsilon), h(\varepsilon), L(\varepsilon))$

$$
=\left(\begin{array}{cc}
L^{2}+\frac{\partial^{2}}{\partial x^{2}}-3 L^{2} A^{2}(\varepsilon)+L^{2} h(\varepsilon) & L^{2} A(\varepsilon) \\
2 \alpha L^{2}\left(2 \frac{\partial A(\varepsilon)}{\partial x} \frac{\partial}{\partial x}+\frac{\partial^{2} A(\varepsilon)}{\partial x^{2}}+A(\varepsilon) \frac{\partial^{2}}{\partial x^{2}}\right) & -L^{2} \frac{\partial^{2}}{\partial x^{2}}-\frac{\partial^{4}}{\partial x^{4}}
\end{array}\right) .
$$

Substituting (85) to (84), we get

$$
\begin{aligned}
\frac{\partial^{2}}{\partial x^{2}} \phi & (\varepsilon)+L^{2} \phi(\varepsilon)-3 L^{2} A^{2}(\varepsilon) \phi(\varepsilon)+L^{2} h(\varepsilon) \phi(\varepsilon) \\
& +L^{2} A(\varepsilon) \psi(\varepsilon)-\lambda(\varepsilon) \phi(\varepsilon)=0, \\
2 \alpha L^{2} & \left(2 \frac{\partial A(\varepsilon)}{\partial x} \frac{\partial \phi(\varepsilon)}{\partial x}+\frac{\partial^{2} A(\varepsilon)}{\partial x^{2}} \phi(\varepsilon)+A(\varepsilon) \frac{\partial^{2} \phi(\varepsilon)}{\partial x^{2}}\right) \\
& -L^{2} \frac{\partial^{2} \psi}{\partial x^{2}}-\frac{\partial^{4} \psi}{\partial x^{4}}-\lambda(\varepsilon) \psi(\varepsilon)=0 .
\end{aligned}
$$

On the branches given in (83), equating the coefficient of $\varepsilon$ in (86) to 0 , we obtain

$$
\begin{gathered}
\phi_{1}^{\prime \prime}+\pi^{2} \phi_{1}-c \pi^{2} \cos \pi x-\lambda_{1} \cos \pi x=0, \\
4 \alpha \pi^{4} \cos 2 \pi x+\pi^{2} \psi_{1}^{\prime \prime}+\psi_{1}^{\prime \prime \prime \prime}+\lambda_{1} c=0 .
\end{gathered}
$$


From (87) and the boundary conditions

$$
\begin{aligned}
& \phi_{1}^{\prime}(0)=\phi_{1}^{\prime}(1)=\phi_{1}^{\prime \prime \prime}(0)=\phi_{1}^{\prime \prime \prime}(1), \\
& \psi_{1}^{\prime}(0)=\psi_{1}^{\prime}(1)=\psi_{1}^{\prime \prime \prime}(0)=\psi_{1}^{\prime \prime \prime}(1),
\end{aligned}
$$

we get

$$
\begin{aligned}
& c=0, \\
& \lambda_{1}=-c \pi^{2}=0 \text {, } \\
& \left(\begin{array}{l}
\phi_{1} \\
\psi_{1}
\end{array}\right)=\left(\begin{array}{c}
\widetilde{\widetilde{p_{1}}} \cos \pi x \\
\underset{\widetilde{q_{1}}}{\widetilde{\widetilde{q_{2}}}} \cos \pi x-\frac{\alpha}{3} \cos 2 \pi x
\end{array}\right) \cdot \widetilde{\widetilde{p_{1}}}, \\
& \widetilde{\widetilde{q_{1}}}, \widetilde{\widetilde{q_{2}}} \in R \text {. }
\end{aligned}
$$

We find that $c=0$ is a contradiction to $c \neq 0$, so there are no solution branches of (4) bifurcated from the trivial solution in Case 3.

Case 4. $c \neq 0, \alpha_{1}=0$.

If $c \neq 0, \alpha_{1}=0$, then $\alpha_{2}= \pm 1$; taking the sign of $\varepsilon$ into account in (15), we consider $\alpha_{2}=1$ only. In this case, from (27), (31), and (37), we have

$$
a_{0}(x)=-2 c c_{1} \cos \pi x, \quad b_{0}(x)=c_{1}, \quad \gamma(0)=0 .
$$

Similarly discussed as in Case 1 previously, we get

$$
\gamma_{1}(0)=\gamma_{2}(0)=\gamma_{3}(0)=\gamma_{4}(0)=\cdots=0 .
$$

Remark 5. Since $\gamma(0)=\gamma_{1}(0)=\gamma_{2}(0)=\gamma_{3}(0)=\gamma_{4}(0)=$ $\cdots=0$, there were no solution branches of (4) bifurcated from the trivial solution in Case 4.

Remark 6. In this paper, trivial stationary solution and bifurcations at it are considered for the KS-GL equations with Neumann's boundary conditions on a finite domain $(0, L)$. The results we get in this paper are new and original. It would be very interesting and much more complicated to try and extend our study to stationary patterns and the corresponding bifurcations. Another interesting but challenging direction would be the case in several space dimensions.

\section{Acknowledgments}

The work is supported by the Youth Science and Technology Innovation Fund of Nanjing Agriculture University (no. KJ2010026), the College Graduate Student Scientific Research Innovation Plan of Jiangsu Province (CXLX12_0390) and the Fundamental Research Funds for the Central Universities (no. Y0201100265).

\section{References}

[1] P. C. Matthews and S. M. Cox, "One-dimensional pattern formation with Galilean invariance near a stationary bifurcation," Physical Review E, vol. 62, no. 2, pp. R1473-R1476, 2000.
[2] P. C. Matthews and S. M. Cox, "Pattern formation with a conservation law," Nonlinearity, vol. 13, no. 4, pp. 1293-1320, 2000.

[3] J. H. P. Dawes, "Localized pattern formation with a large-scale mode: slanted snaking," SIAM Journal on Applied Dynamical Systems, vol. 7, no. 1, pp. 186-206, 2008.

[4] A. A. Golovin, A. A. Nepomnyashchy, and L. M. Pismen, "Interaction between short-scale Marangoni convection and long-scale deformational instability," Physics of Fluids, vol. 6, no. 1, pp. 34-48, 1994.

[5] L. Hocking, K. Stewartson, and J. Stuart, "A nonlinear instability burst in plane parallel flow," Journal of Fluid Mechanics, vol. 51, no. 4, pp. 705-735, 1972.

[6] C. R. Doering, J. D. Gibbon, D. D. Holm, and B. Nicolaenko, "Low-dimensional behaviour in the complex Ginzburg-Landau equation," Nonlinearity, vol. 1, no. 2, pp. 279-309, 1988.

[7] B. Nicolaenko, B. Scheurer, and R. Temam, "Some global dynamical properties of the Kuramoto-Sivashinsky equations: nonlinear stability and attractors," Physica D, vol. 16, no. 2, pp. 155-183, 1985.

[8] D. Kazhdan, L. Shtilman, A. A. Golovin, and L. M. Pismen, "Nonlinear waves and turbulence in Marangoni convection," Physics of Fluids, vol. 7, no. 11, pp. 2679-2685, 1995.

[9] J. Q. Duan, C. Bu, H. J. Gao, and M. Taboada, "On a coupled Kuramoto-Sivashinsky and Ginzburg-Landau-type model for the Marangoni convection," Journal of Mathematical Physics, vol. 38, no. 5, pp. 2465-2474, 1997.

[10] Q. K. Xiao and H. J. Gao, "Bifurcation analysis of the SwiftHohenberg equation with quintic nonlinearity," International Journal of Bifurcation and Chaos, vol. 19, no. 9, pp. 2927-2937, 2009.

[11] C. P. Li and G. Chen, "Bifurcation analysis of the KuramotoSivashinsky equation in one spatial dimension," International Journal of Bifurcation and Chaos, vol. 11, no. 9, pp. 2493-2499, 2001.

[12] C. P. Li and G. Chen, "Bifurcation from an equilibrium of the steady state Kuramoto-Sivashinsky equation in two spatial dimensions," International Journal of Bifurcation and Chaos, vol. 12, no. 1, pp. 103-114, 2002. 


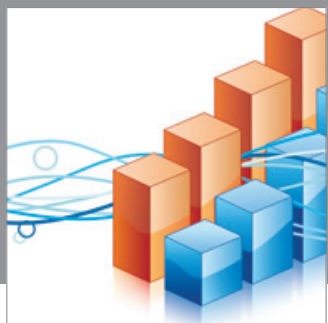

Advances in

Operations Research

mansans

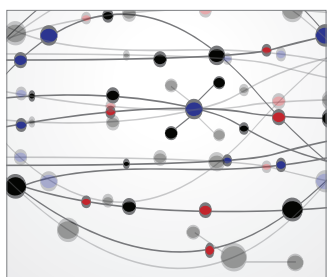

The Scientific World Journal
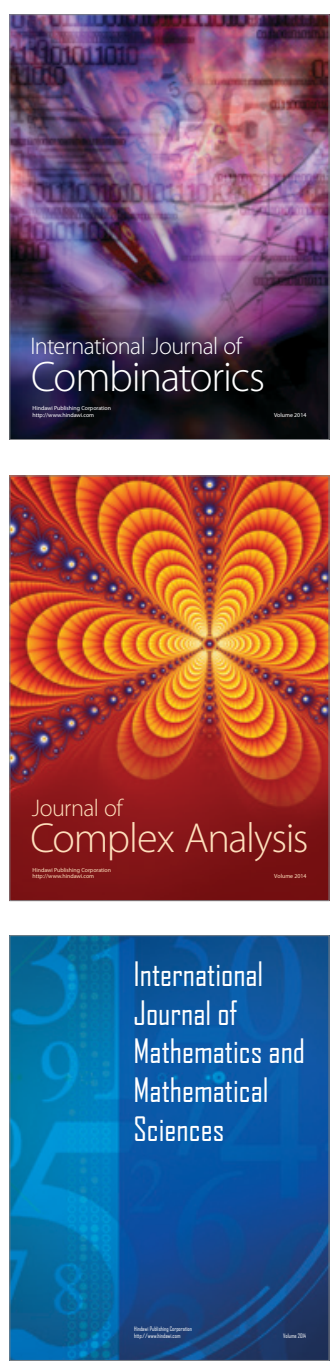
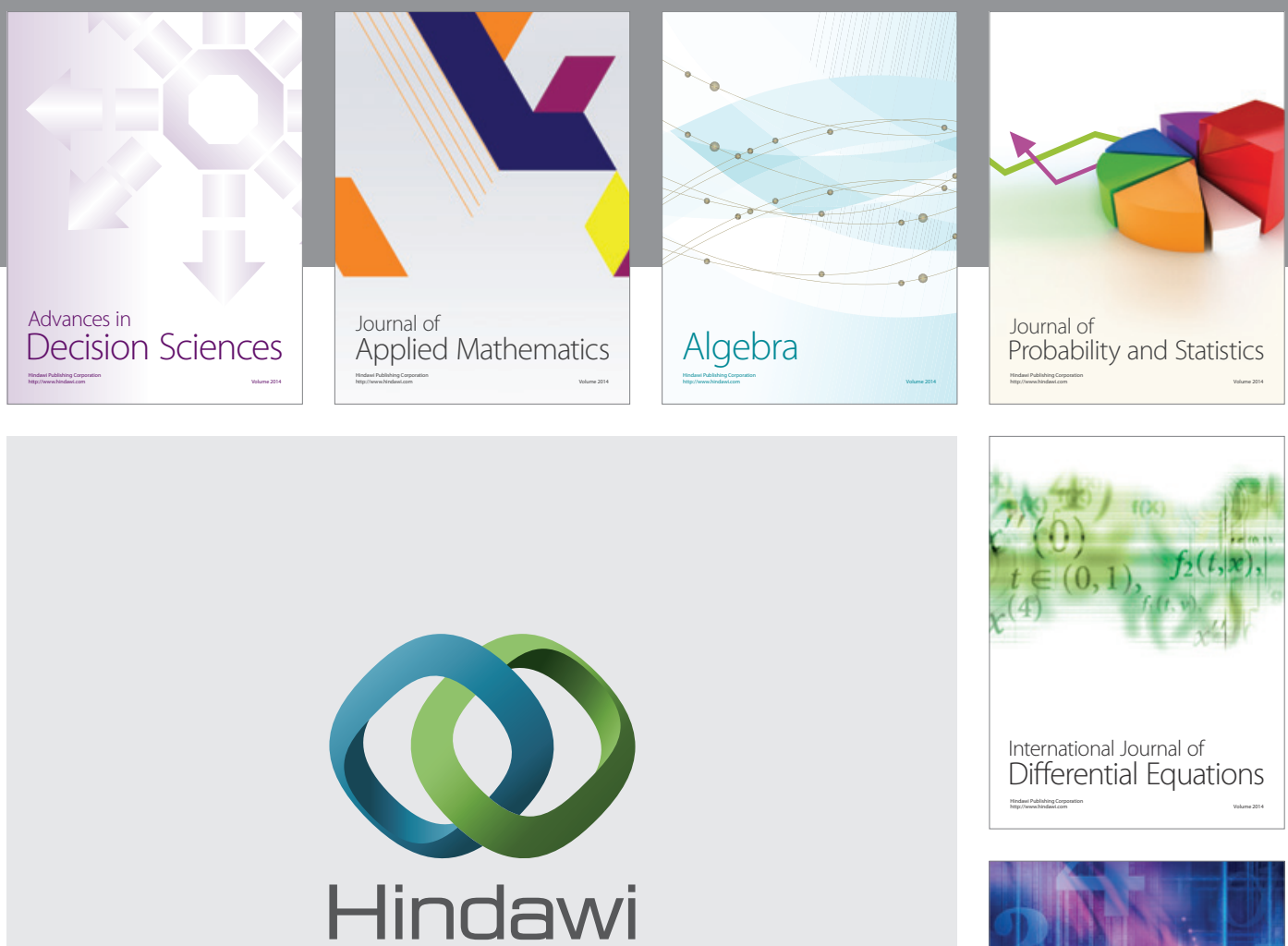

Submit your manuscripts at http://www.hindawi.com
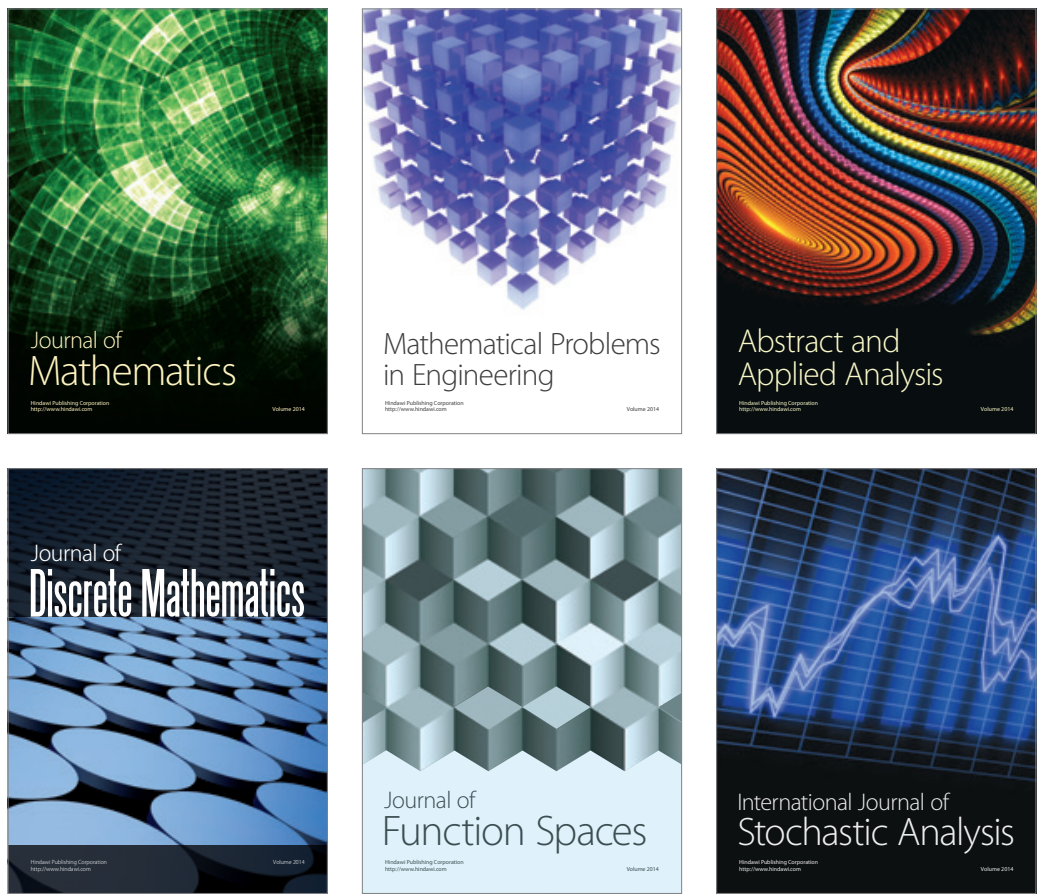

Journal of

Function Spaces

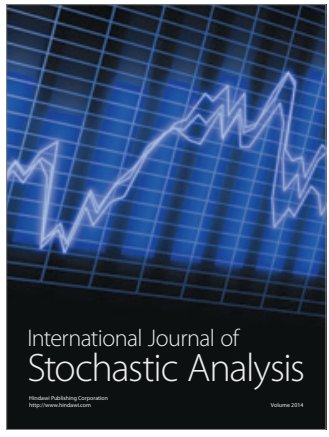

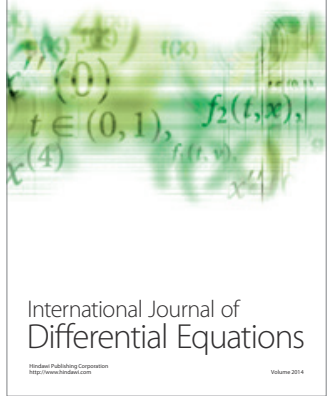
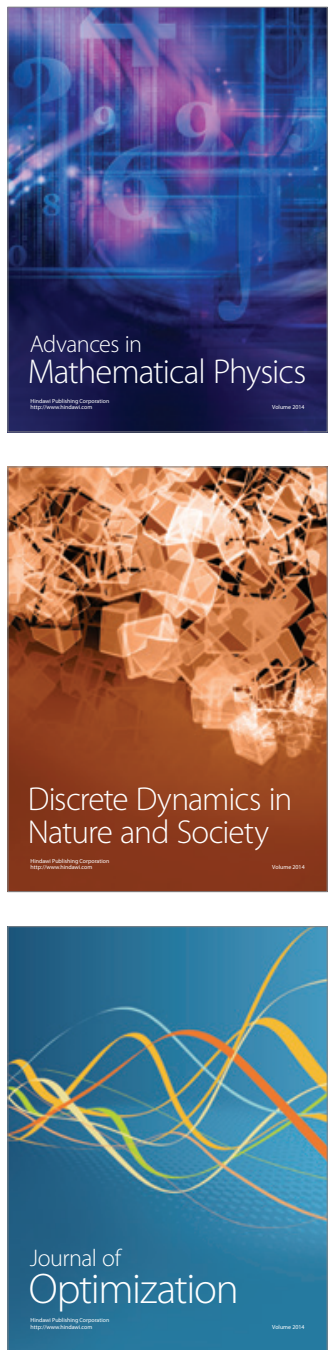